\title{
Winning abstracts selected through 999 EMS Research Forum peer review process and presented orally or by poster at Ambex 2004
}

\section{WINNER OF THE EMERGENCY MEDICINE JOURNAL (EMJ) AWARDED FOR MOST ORIGINAL RESEARCH}

001 10\% OR 50\% DEXTROSE IN THE TREATMENT OF HYPOGLYCAEMIA OUT-OF-HOSPITAL? A RANDOMISED CONTROLLED TRIAL

C. Moore, M. Woollard. Pre-hospital Emergency Research Unit, Welsh Ambulance Services NHS Trust, Lansdowne Hospital, Cardiff, UK

Objective: This randomised controlled trial aimed to determine whether $10 \%$ dextrose is as safe and effective as $50 \%$ dextrose when treating hypoglycaemia out-of-hospital.

Methods: Hypoglycaemic patients in the out-of-hospital setting with a blood glucose level of $4 \mathrm{mmols} / \mathrm{l}$ or less were randomised to receive either intravenous $10 \%$ or $50 \%$ dextrose. Paramedics in a large UK ambulance service administered either concentration of dextrose in 5gram increments. $10 \%$ dextrose was administered in $50 \mathrm{ml}$ aliquots via a three-way tap and giving set and $50 \%$ in $10 \mathrm{ml}$ aliquots from pre-filled $(25 \mathrm{~g}$ in $50 \mathrm{ml})$ syringes. The maximum cumulative dose for subjects in both groups was limited to $25 \mathrm{~g}$. Paramedics recorded standard pre and post-treatment clinical data and rated the convenience of administration of each dextrose formulation using a lickert scale.

Results: Fifty one patients were recruited $(10 \% n=25,50 \% n=26)$. There were no significant differences between groups in age or sex profile, median pretreatment Glasgow Coma Score (GCS) and blood glucose level, or the proportion of insulin dependent diabetics. Following treatment there were no significant differences in median time to recovery (8 minutes), median post-treatment GCS (15), subjects experiencing a further hypoglycaemic episode within 24 hours (4 per group), time on scene, or ease of administration. There were, however, significant differences in the median total dose of dextrose administered $110 \%=$ 10 grams, $50 \%=25$ grams, $\mathrm{p}<0.001$ ) and median post-treatment blood sugar levels $(10 \%=6.2 \mathrm{mmols} / \mathrm{l}$ and $50 \%=9.4 \mathrm{mmols} / \mathrm{l}, \mathrm{p}=0.003)$. There were no reports of extravasation injury from either group.

Conclusions: $10 \%$ dextrose proved as effective as $50 \%$ in the out-ofhospital treatment of hypoglycaemia. It is administered in lower doses than a $50 \%$ presentation, which results in more acceptable posttreatment blood glucose levels.

\section{WINNER OF THE EMERGENCY MEDICINE JOURNAL} (EMJ) AWARDED FOR BEST POSTER

005 USE OF AN ACTIVE COMPRESSION DECOMPRESSION DEVICE IN PRE HOSPITAL CARDIAC ARREST BY AMBULANCE PARAMEDICS AND AMBULANCE TECHNICIANS

M. S. Box. Dorset Ambulance NHS Trust, UK

Introduction: The active compression decompression device the AMBUCARDIO PUMP has just been introduced to the UK prehospital market. This study used the AMBU- CARDIO PUMP to assess the effects of active compression decompression CPR compared with standard CPR in the prehospital environment.

Method: Patients who suffered a cardiac arrest in the Bournemouth conurbation were randomly assigned to receive either active compression decompression CPR or standard CPR depending on which crew attended the arrest. The primary study end point was arrival at a designated A\&E Department, with a secondary end study point of 4 weeks post arrest.

Results: Short term outcome among patients assigned to standard CPR and active compression decompression CPR.

\begin{tabular}{lll} 
Abstract 005 & & \\
\hline & $\begin{array}{l}\text { STANDARD CPR } \\
\mathrm{N}=12\end{array}$ & $\begin{array}{l}\text { ACD CPR } \\
\mathrm{N}=12\end{array}$ \\
\hline Return of spontaneous circulation & $33 \%$ & $66 \%$ \\
Survival at one hour & $33 \%$ & $58 \%$ \\
Admission to i t u & $33 \%$ & $58 \%$ \\
Survival at 24 hours & $8 \%$ & $50 \%$ \\
Survival at 7 days & $8 \%$ & $41 \%$ \\
Survival at 4 weeks & $0 \%$ & $33 \%$ \\
\hline
\end{tabular}

Short term outcome among patients assigned to standard CPR and active compression decompression CPR

Both the ACD-CPR and standard CPR groups ended up with similar numbers in each group, with each group being $66 \%$ male. The initial cardiac rhythm of VF occurred in $41 \%$ of the ACD-CPR group against $58 \%$ for standard CPR. At the primary end study point $66 \%$ of the patients treated with ACD-CPR had return of spontaneous circulation compared to $33 \%$ for standard CPR. Survival at 4 weeks was measured at $33 \%$ for the ACD-CPR with none of the standard CPR group surviving past 7 days

Conclusions: Active compression decompression CPR using the AMBU-CARDIO PUMP performed during advanced life support appears to have significantly improved survival rates among patients who have a prehospital cardiac arrest.

The author recognises that this is small-scale research and the findings would suggest that further research into active compression decompression CPR is indicated.

WINNER OF THE JOINT ROYAL COLLEGES

AMBULANCE LIAISON COMMITTEE (JRCALC)

AWARD FOR RESEARCH MOST LIKELY TO AFFECT

PRACTICE

006 A MULTI-CENTRE EVALUATION OF MANAGING LOW PRIORITY 999 CALLS BY NURSE ADVICE - PROGRESS SO FAR

J. Turner ${ }^{1}$, H. Snooks ${ }^{2}$. 'Medical Care Research Unit, University of Sheffield, UK; ${ }^{2}$ The Clinical School, University of Wales Swansea, UK

Study Aim: The aim of the study is to assess the clinical service and cost effectiveness of transferring some non-urgent 999 ambulance calls to NHS Direct nurse advisers for further assessment and management.

Methods: The study has been designed using a mixed randomised controlled trial (RCT) and controlled observational study approach. There are 2 stages:

Stage 1 - Is a randomised controlled trial. Over a 12 month period individuals calling 999 and triaged by the ambulance control call taker to one of the study EMD codes are randomly allocated to receive further assessment by the nurse adviser or dispatch of the standard response of a paramedic ambulance after consenting to participate. These callers are then sent a postal questionnaire to elicit what action they took and their satisfaction with the service received. Comparisons will be made between intervention and control groups to determine if the service change provides a clinically effective and acceptable service.

Stage 2 - Is a controlled observational study. Over a 4 month period all calls with appropriate EMD codes will be passed to nurse advisers to identify the practical and operational effects of the service change. The cost consequences of the service will also be measured.

Practicalities: The practical implications of setting up and conducting a research trial in an operational environment can be difficult. Strategies to overcome these have included: 
- Obtaining patient consent - Consent has to be obtained verbally at the time of the 999 call. A pre-consent randomisation design has been used to simplify this process and minimise confusion and the amount of explanation required. Simple consent protocols for EMDs and nurses have been developed and a standardised recruitment algorithm provided to each service.

- Randomisation procedures - For Omega calls an electronic randomisation programme has been built in to the AMPDS system. In Manchester, which uses Alpha calls, EMD stations are randomly assigned to intervention or control group each day. EMDs refer identified calls to the appropriate response for that day.

- Developing referral processes - In Manchester a seamless, integrated call transfer process has been developed. The other sites refer to a remote NHS Direct centre where calls are directed straight to a nurse identified to accept ambulance service calls and bypassing the initial NHS Direct call handling process.

- Gaining staff engagement and co-operation in the project - A project board involving the research team, senior managers from both ambulance and NHS Direct services, EMDs and nurses was set up before the project started. All members have been fully involved in setting up and developing the practical components of consent and recruitment procedures, referral processes and data collection. The group meets regularly to review progress, identify problems and share experiences and best practice.

Progress so far: The RCT started in Autumn 2003. The Two Shires, Oxfordshire and Welsh ambulance service are testing AMPDS Omega codes and transfer calls to an NHS Direct call centre for nurse advice. Greater Manchester Ambulance Service are testing AMPDS Alpha codes and nurse advice is provided in the control room.

To date 1583 calls have been randomised to the intervention group and 1847 to the control group. About one third of these calls have given consent for further follow up. A high proportion of AMPDS Alpha calls are returned to the ambulance service $(67 \%)$ whereas only $35 \%$ of Omega calls are returned at this point in the study. 